\title{
Einstein's Equation of motion for exterior test particles wi distribution having varied field, time and radial distance; Golden metric
}

\section{tensors approach.}

International Journal of Theoretical \& Computational Physics

Research Article

U. Maisalatee $^{1 *}$, U. Rilwan ${ }^{2}$ and E.I. Ugwu ${ }^{2}$

*Correspondence author

${ }^{1}$ Liyu Unity Science Academy, Campus Avenue, Behind Yaro Sule Filling Station, PMB 03 Keffi, Nasarawa State.

U. Rilwan

Liyu Unity Science Academy

Campus Avenue

Behind Yaro Sule Filling Station

${ }^{2}$ Nigerian Army University, PMB. 1500, Biu, Borno State.

PMB 03 Keffi

Nasarawa State

Submitted : 16 Jun 2021 ; Published : 3 Jul 2021

\begin{abstract}
Golden metric tensors exterior to hypothetical distribution of mass whose field varies with time and radial distance have been used to construct the coefficient of affine connections that invariably was used to obtained the Einstein's equations of motion for test particles of non-zero rest masses. The expression for the variation of time on a clock moving in this gravitational field was derived using the time equation of motion. The test particles in this field under the condition of pure polar motion have an inverse square dependence velocity which depends on radial distance. Our result indicates that despite using the golden metric tensor, the inverse square dependence of the velocity on radial distance has not been changed.
\end{abstract}

Keywords: Golden Metric Tensors, Gravitational Field, Time, Radial Distance, Test Particles.

\section{Introduction}

The geometrical theory of gravitation was first published by Albert Einstein in 1915/16 known as General Relativity. The most widely accepted theory gravitation is the General Relativity $[1,2]$.

The search for the solutions both exact and analytical has been an ongoing research [1-4]. The exact solution to this theory of gravitation was first constructed in pure radial and static spherical polar coordinates in 1916 by Schwarzschild. In Schwarzschild's metric, the tensor field varies with radial distance only. It is the metric tensor exterior to an ideal static spherically symmetric body situated in empty space [5-9]. Schwarzschild's metric is the mathematically simplest and most satisfactory astrophysical solution of Einstein's geometrical gravitational field equation in the space exterior to a static homogeneous distribution of mass within a spherical region [6]

Einstein's equations are the centre piece of general relativity. They provide a precise formation of the relation between space time geometry and the properties of matter, using mathematics. More concretely, they are formulated using the concept of Riemannian geometry, in which the geometric properties of a space time are described by a quantity called a metric. The metric encodes the information needed to compute the fundamental geometric notations distance and angle in a curved space [10].
In general relativity, the metric and the Riemann curvature tensor are quantities define at each point in space time. It is based on this that Howusu by postulation introduced a second natural and satisfactorily generalization or extension of the Schwarzschild's metric tensor from the gravitational field of all static homogeneous spherical distribution of mass to the gravitational fields of all spherical distributions of mass named as the golden metric tensor for all gravitational field in nature [10-11].

Research has shown that spherical systems doesn't depend on radial distance only [2], therefore in this paper we introduced an astrophysical system within the region of spherical geometry, whose tensor fields varies with radial distance and time, using Golden metric tensors. The coefficients of affine connections for this field were constructed and used in the study of Einstein's equations of motion for test particles of non-zero rest masses.

\section{Golden metric tensors}

The covariant golden metric tensors are given by [10, 12-13]

$$
\begin{aligned}
& g_{11}=\left[1+\frac{2}{c^{2}} f(t, r)\right]^{-1} . \\
& g_{22}=r^{2}\left[1+\frac{2}{c^{2}} f(t, r)\right]^{-1} .
\end{aligned}
$$


$g_{33}=r^{2} \sin ^{2} \theta\left[1+\frac{2}{c^{2}} f(t, r)\right]^{-1}$.

$g_{00}=-\left[1+\frac{2}{c^{2}} f(t, r)\right]$

$g_{u v}=0$.

While the contravariant metric tensors are given by $[10,12-13]$

$$
\begin{aligned}
& g^{11}=\left[1+\frac{2}{c^{2}} f(t, r)\right] . \\
& g^{22}=\frac{1}{r^{2}}\left[1+\frac{2}{c^{2}} f(t, r)\right] . \\
& g^{33}=\frac{1}{r^{2} \sin ^{2} \theta}\left[1+\frac{2}{c^{2}} f(t, r)\right] . \\
& g^{00}=-\left[1+\frac{2}{c^{2}} f(t, r)\right]^{-1} . \\
& g^{\mu v}=0 .
\end{aligned}
$$

The coefficients of affine connections [2, 6, 12, 14-15] defined by metric tensors of space time are generally given as

$$
\Gamma_{\alpha \beta}^{\gamma}=\frac{1}{2} g^{\gamma \xi}\left(g_{\alpha \xi, \beta}+g_{\beta \xi, \alpha}-g_{\alpha \beta, \xi}\right) .
$$

Using the above metric tensors (1)-(10), the coefficient of affine connections are given as

$$
\begin{aligned}
& \Gamma_{00}^{0}=\frac{1}{2} g^{00} g_{00,0} . \\
& \Gamma_{01}^{0}=\Gamma_{10}^{0}=\frac{1}{2} g^{00} g_{00,1} . \\
& \Gamma_{11}^{0}=-\frac{1}{2} g^{00} g_{11,0} . \\
& \Gamma_{00}^{1}=-\frac{1}{2} g^{11} g_{00,1} . \\
& \Gamma_{10}^{1}=\Gamma_{01}^{1}=\frac{1}{2} g^{11} g_{11,0} . \\
& \Gamma_{11}^{1}=\frac{1}{2} g^{11} g_{11,1} . \\
& \Gamma_{22}^{1}=-\frac{1}{2} g^{11} g_{22,1} . \\
& \Gamma^{1}{ }_{12}=-\frac{1}{2} g^{11} g_{33,1} . \\
& \Gamma_{21}=\frac{1}{2} g^{22} g_{22,1} .
\end{aligned}
$$

$$
\begin{aligned}
& \Gamma_{13}^{3}=\Gamma_{31}^{3}=\frac{1}{2} g^{33} g_{33,1} . \\
& \Gamma_{\alpha \beta}^{\mu}=0,
\end{aligned}
$$

Equations (12) -(22) are given explicitly as

$$
\Gamma_{00}^{0}=\frac{1}{c^{2}}\left[1+\frac{2}{c^{2}} f(t, r)\right]^{-1} \frac{\partial f(t, r)}{\partial t} .
$$

$\Gamma_{01}^{0}=\Gamma_{10}^{0}=\frac{1}{c^{2}}\left[1+\frac{2}{c^{2}} f(t, r)\right]^{-1} \frac{\partial f(t, r)}{\partial r}$.

$\Gamma_{11}^{0}=-\frac{1}{c^{2}}\left[1+\frac{2}{c^{2}} f(t, r)\right]^{-3} \frac{\partial f(t, r)}{\partial t}$.

$\Gamma_{00}^{1}=\frac{1}{c^{2}}\left[1+\frac{2}{c^{2}} f(t, r)\right] \frac{\partial f(t, r)}{\partial r}$.

$\Gamma_{10}^{1}=\Gamma_{01}^{1}=-\frac{1}{c^{2}}\left[1+\frac{2}{c^{2}} f(t, r)\right]^{-1} \frac{\partial f(t, r)}{\partial t}$.

$\Gamma_{11}^{1}=-\frac{1}{c^{2}}\left[1+\frac{2}{c^{2}} f(t, r)\right]^{-1} \frac{\partial f(t, r)}{\partial r}$.

$\Gamma_{22}^{1}=-r+\frac{r^{2}}{c^{2}}\left[1+\frac{2}{c^{2}} f(t, r)\right]^{-1} \frac{\partial f(t, r)}{\partial r}$.

$\Gamma_{33}^{1}=-r \sin ^{2} \theta+\frac{r^{2} \sin ^{2} \theta}{c^{2}}\left[1+\frac{2}{c^{2}} f(t, r)\right]^{-1} \frac{\partial f(t, r)}{\partial r}$.

$\Gamma_{12}^{2}=\Gamma_{21}^{2}=\frac{1}{r}-\frac{1}{c^{2}}\left[1+\frac{2}{c^{2}} f(t, r)\right]^{-1} \frac{\partial f(t, r)}{\partial r}$.

$\Gamma_{13}^{3}=\Gamma_{31}^{3}=\frac{1}{r}-\frac{1}{c^{2}}\left[1+\frac{2}{c^{2}} f(t, r)\right]^{-1} \frac{\partial f(t, r)}{\partial r}$.

$\Gamma_{\alpha \beta}^{\mu}=0$, otherwise

Using the Golden metric tensors, we have obtained 10 nonzero coefficients of affine connections unlike the 9 obtained in Schwarzschild field. Based on this fact, this gravitational field is expected to have some additional terms not found in Schwartzschild's field.

Motion of test particles exterior to spherical bodies whose fields varies with time and radial distance

The general relativistic equation of motion for test particles in a gravitational field is given by $[2,6,10,12,16-17]$ 


$$
\frac{d^{2} x^{\mu}}{\partial \tau^{2}}+\Gamma_{\alpha \beta}^{\mu}\left[\frac{d x^{\alpha}}{d \tau}\right]\left[\frac{d x^{\beta}}{d \tau}\right]=0 .
$$

where $\tau$ is the proper time

Setting $\mu=0$ into (34) gives

$i+\frac{1}{c^{2}}\left[1+\frac{2}{c^{2}} f(t, r)\right]^{-1} \frac{\partial f(t, r)}{\partial t} t^{2}+\frac{2}{c^{2}}\left[1+\frac{2}{c^{2}} f(t, r)\right]^{-1} \frac{\partial f(t, r)}{\partial r} t r-\frac{1}{c^{2}}\left[1+\frac{2}{c^{2}} f(t, r)\right]^{-3} \frac{\partial f(t, r)}{\partial t} \dot{r}^{2}=0$.

Simplifying (35) gives

$$
\frac{d}{d \tau}\left[\ln \dot{t}+\ln \left[1+\frac{2}{c^{2}} f(t, r)\right]\right]=0
$$

Integrating (36) gives

$$
\dot{t}=A\left[1+\frac{2}{c^{2}} f(t, r)\right]^{-1} .
$$

As $\mathrm{t} \rightarrow \tau, f(t, r) \rightarrow 0$ and the constant $A \equiv 1$,

$$
\dot{t}=\left[1+\frac{2}{c^{2}} f(t, r)\right]^{-1} \text {. }
$$

Setting $\mu=1,2,3$ into (34) gives

$r+\frac{1}{c^{2}}\left[1+\frac{2}{c^{2}} f(t, r)\right] \frac{\partial f(t, r)}{\partial r} t^{2}-\frac{2}{c^{2}}\left[1+\frac{2}{c^{2}} f(t, r)\right]^{-1} \frac{\partial f(t, r)}{\partial t} t r-\frac{1}{c^{2}}\left[1+\frac{2}{c^{2}} f(t, r)\right]^{-1} \frac{\partial f(t, r)}{\partial r} \dot{r}^{2}+$

$\left[-r+\frac{r^{2}}{c^{2}}\left[1+\frac{2}{c^{2}} f(t, r)\right]^{-1} \frac{\partial f(t, r)}{\partial r}\right] \dot{\theta}^{2}+\left[-r \sin ^{2} \theta+\frac{r^{2} \sin ^{2} \theta}{c^{2}}\left[1+\frac{2}{c^{2}} f(t, r)\right]^{-1} \frac{\partial f(t, r)}{\partial r}\right] \dot{\phi}^{2}=0$

For pure radial motion $\theta=\varnothing=0$

$\ddot{r}+\frac{1}{c^{2}}\left[1+\frac{2}{c^{2}} f(t, r)\right] \frac{\partial f(t, r)}{\partial r} t^{2}-\frac{2}{c^{2}}\left[1+\frac{2}{c^{2}} f(t, r)\right]^{-1} \frac{\partial f(t, r)}{\partial t} t r-\frac{1}{c^{2}}\left[1+\frac{2}{c^{2}} f(t, r)\right]^{-1} \frac{\partial f(t, r)}{\partial r} \dot{r}^{2}=0$

$\ddot{\theta}+2\left[\frac{1}{r}-\frac{1}{c^{2}}\left[1+\frac{2}{c^{2}} f(t, r)\right]^{-1} \frac{\partial f(t, r)}{\partial r}\right] \dot{r} \dot{\theta}=0$.

$\ddot{\phi}+2\left[\frac{1}{r}-\frac{1}{c^{2}}\left[1+\frac{2}{c^{2}} f(t, r)\right]^{-1} \frac{\partial f(t, r)}{\partial r}\right] \dot{r} \dot{\phi}=0$.

$\frac{\ddot{\phi}}{\dot{\phi}}+2\left[\frac{1}{r}-\frac{1}{c^{2}}\left[1+\frac{2}{c^{2}} f(t, r)\right]^{-1} \frac{\partial f(t, r)}{\partial r}\right] \dot{r}=0$.

To the limit of $c^{\circ}(43)$ reduces to

$$
\frac{\ddot{\phi}}{\dot{\phi}}+\frac{2}{r} \dot{r}=0 .
$$

Integrating (44) yields

$$
\dot{\phi}=\frac{A}{r^{2}}
$$

where $\mathrm{A}$ is constant of integration.

This motion has an inverse square dependence on the radial distance. Therefore the golden metric tensor to the limit of does not change the inverse square dependence on the radial distance.

\section{Conclusion}

The coefficients of affine connections for the first time are constructed for this field using Golden metric tensors.

The time, radial, polar and azimuthal equation of motion for test particles exterior to astrophysical distribution of mass were given in equation (35), (39), (41) and (42) respectively. The solution of the time equation of motion (38) gives the variation of the time on a clock within the gravitational field, which is the equation for the time dilation within this gravitational field.

Despite using Golden metric tensors our expression for the time equation of motion is the same with that obtained by Lumbi et al., (2014). The only difference is that in Lumbi et al., the gravitational scalar potential is a function of radial time, distance and polar angle while our gravitational scalar potential is a function of time and radial distance only.

Equation (40) which is the radial equation of motion can be used to obtain the instantaneous speed of a particle of non-zero rest mass in this field.

The coefficient of affine connections obtained in this work can be used to construct the Lagrangian equation of motion, the Riemann-Christoffel, Ricci and Einstein's tensors as well as Einstein's geometrical field equations for this field, the field equation would only contain a single unknown value $f(t, r)$ which can be solved completely to obtain an explicit value for $f(t, r)$

\section{References}

1. E.N. Chifu (2012) Gravitational fields exterior to a homogeneous spherical masses. The Abraham Zelmanov Journal. 2012, 5: 31-67.

2. A.U. Maisalatee, M.M. Azos\& I.I. Ewa (2021) Complete Einstein's equation of motion for test particles exterior to spherical massive bodies using a varying potential. International Astronomy and Astrophysics Research Journal. 2021, 3(1): 43-53.

3. S.X.K. Howusu (2010) Einstein's Geometrical Field Equations, Jos University Press Ltd, 2010:34.

4. E.N. Chifu, S.X.K.Howusu (2009) Solution of Einstein's geometrical gravitational field equations exterior to astrophysically real or hypothetical time varying distributions of mass within regions of spherical geometry. Progress in Physics. 2009, 3: 45-48.

5. A.U. Maisalatee, E.N. Chifu, W.L. Lumbi, M.U. Sarki, and M.Mohammed (2020) Solution of Einstein's field equation exterior to a spherical mass with varying potential. Dutse Journal of Pure and Applied Sciences (DUJOPAS). 2020, 6(2): 294-301.

6. L.W. Lumbi, I.I. Ewa, N.Tsaku (2014) Einstein's equations of motion for test particles exterior to spherical distributions of mass whose tensor field varies with time, 
radial distance and polar angle. Archives of Applied Science Research Library. 2014, 6(5): 36-41.

7. E.N.Chifu, S.X.K.Howusu and W.L.Lumbi. (2009) Relativistic mechanics in gravitational fields exterior to rotating homogeneous mass distributions within regions of spherical geometry. Progress in Physics, 2009, 3:18-23.

8. S.X.K. Howusu (2007) National Philosophy Society, Jos. 2007: 2-227.

9. K. Schwarzschild (2008) Abraham Zelmanov Journal, 2008, 1:10-19.

10. Y. Nura, S.X.K. Howusu, L.W. Lumbi, I. Nuhu, and A. Hayatu (2017) International Journal of Theoretical and Mathematical Physics. 2017, 7(2): 25-35.

11. S.X.K. Howusu (1991) On the gravitation of moving bodies. Physics Essay. 1991, 4(1): 81-93.

12. S. X. K.Howusu (2009) The Metric Tensors for Gravitational Fields and The Mathematical Principles of Riemann Theoretical Physics, Jos University Press Ltd., 2009: 19-122.

13. S. X. K.Howusu (2011) Riemannian Resolution in Mathematics and Physics. Jos University Press Ltd., 2011: iv.

14. G.Arfken (1995) Mathematical Method for Physicists, $5^{\text {th }}$ edition, Academic Press, New York. 1995: 233.

15. P.G.Bergmann (1987) Introduction to the Theory of Relativity, Prentice Hall, India. 1987:203.

16. Chifu EN, Lumbi WL (2008) General relativistic equations of motion for test particles exterior to astrophysically real or hypothethetically spherical distribution of mass whose tensor field varies with azimuthal angle only. Continental J. Applied Sciences. 2008, 3(8): 32-38.

17. K.Peter, S.Dunsby (2000) An Introduction to Tensors and Relativity; Cape Town. 2000: 51-110.

Copyright: (C2021 U. Rilwan. This is an open-access article distributed under the terms of the Creative Commons Attribution License, which permits unrestricted use, distribution, and reproduction in anymedium, provided the original author and source are credited. 